\title{
Accelerated Exponentially Fitted Operator Method for Singularly Perturbed Problems with Integral Boundary Condition
}

\author{
Habtamu Garoma Debela (iD and Gemechis File Duressa \\ Department of Mathematics, College of Natural Sciences, Jimma University, Jimma, Ethiopia \\ Correspondence should be addressed to Habtamu Garoma Debela; habte200@gmail.com
}

Received 4 December 2019; Accepted 17 February 2020; Published 16 March 2020

Academic Editor: Jian-Ping Sun

Copyright (C) 2020 Habtamu Garoma Debela and Gemechis File Duressa. This is an open access article distributed under the Creative Commons Attribution License, which permits unrestricted use, distribution, and reproduction in any medium, provided the original work is properly cited.

In this paper, we consider a class of singularly perturbed differential equations of convection diffusion type with integral boundary condition. An accelerated uniformly convergent numerical method is constructed via exponentially fitted operator method using Richardson extrapolation techniques and numerical integration methods to solve the problem. The integral boundary condition is treated using numerical integration techniques. Maximum absolute errors and rates of convergence for different values of perturbation parameter and mesh sizes are tabulated for the numerical example considered. The method is shown to be $\varepsilon$-uniformly convergent.

\section{Introduction}

Boundary value problems involving integral boundary conditions have received considerable attention in recent years $[1,2]$. For a discussion of existence and uniqueness results and for applications of problems with integral boundary conditions, one can refer [3-5] and the references therein. In [2, 6, 7], some approximating or numerical treatment aspects of this kind of problems have been considered. However, the methods or algorithms developed so far mainly concerned with the regular cases (i.e., when the boundary layers are absent). Boundary value problems with integral boundary conditions in which the leading derivative term is multiplied by a small parameter are called singularly perturbed problems with integral boundary conditions. The solutions of such types of problems manifest boundary layer phenomena where the solution changed abruptly. As a result, numerical analysis of singular perturbation cases has been far from trivial because of the boundary layer behavior of the solution. The solutions of the problems with boundary layer undergo rapid changes within very thin layers near the boundary or inside the problem domain $[2,8-10]$, and hence classical numerical methods for solving such problems are unstable and fail to give good results when the perturbation parameter is small (i.e., for $h \geq \varepsilon$ ) [10]. Therefore, it is important to develop a numerical method that gives good results for small values of the perturbation parameter where others fails to give good result and convergent independent of the values of the perturbation parameter. In recent years, the authors [11-15] have developed various numerical schemes on uniform meshes for singularly perturbed first and second order differential equations with integral boundary conditions.

As far as the researchers' knowledge is concerned numerical solution of the singularly perturbed boundary value problem containing integral boundary condition via the accelerated exponential fitted operator method is first being considered. Hence, this paper proposed a uniformly convergent numerical method based on exponential fitted operator and numerical integration methods to solve the problem under consideration.

\section{Statement of the Problem}

Consider the following singularly perturbed problem with integral boundary condition:

$$
\begin{aligned}
L y & \equiv \varepsilon^{2} y^{\prime \prime}(x)+\varepsilon a(x) y^{\prime}(x)-b(x) y(x)=f(x), \quad 0<x<l, \\
y(0) & =\mu_{0}, \\
L_{1} y & =y(l)-\int_{l_{0}}^{l_{1}} g(x) y(x) \mathrm{d} x=\mu_{1}, \quad 0 \leq l_{0}<l_{1} \leq l,
\end{aligned}
$$


where $\varepsilon, 0<\varepsilon \ll 1$ is a perturbation parameter, $\mu_{0}$ and $\mu_{1}$ are given constants, and the functions $a(x) \geq \alpha>0, b(x) \geq \beta>0, g(x)$, and $f(x)$ are sufficiently smooth functions in the $[0, l]$. The solution $y(x)$ of problems (1)-(3) has in general boundary layers at $x=0$ and $x=l$ for $\varepsilon$ near 0 .

In this paper, we analyze the exponential fitted operator scheme with numerical integration techniques on a uniform mesh for the numerical solution of equations (1)-(3). Uniform convergence is proved in the discrete maximum norm. Finally, we formulate the algorithm for solving the discrete problem and give the illustrative numerical results.

\section{Properties of Continuous Solution}

The differential operator for equation (1) is given by

$$
L_{\varepsilon} \equiv \varepsilon^{2} \frac{\mathrm{d}^{2}}{\mathrm{~d} x^{2}}+\varepsilon a \frac{\mathrm{d}}{\mathrm{d} x}-b,
$$

and it satisfies the following minimum principle for boundary value problems (BVPs). The following lemmas [9] are necessary for the existence and uniqueness of the solution and for the problem to be well posed.

Lemma 1 (continuous minimum principle). Assume that $v(x)$ is any sufficiently smooth function satisfying $v(0) \geq 0$ and $v(l) \geq 0$. Then, $\operatorname{Lv}(x) \leq 0$ for all $x \in \Omega=(0, l)$ implies that $v(x)>0$ for all $x \bar{\epsilon} \Omega=[0, l]$.

Proof. Let $x^{*}$ be such that $v\left(x^{*}\right)=\min _{x \in[0, l]} v(x)$ and assume that $v\left(x^{*}\right)<0$. Clearly $x^{*} \notin\{0, l\}$; therefore, $v^{\prime}\left(x^{*}\right)=0 \quad$ and $\quad v^{\prime \prime}\left(x^{*}\right) \geq 0$. Moreover, $L v\left(x^{*}\right)=\varepsilon^{2} v^{\prime \prime}\left(x^{*}\right)+\varepsilon a\left(x^{*}\right) v^{\prime}\left(x^{*}\right)-b\left(x^{*}\right) v\left(x^{*}\right) \geq 0$, which is a contradiction. It follows that $v\left(x^{*}\right) \geq 0$ and thus $v(x) \geq 0, \forall x \in[0, l]$.

The uniqueness of the solution is implied by this minimum principle. Its existence follows trivially (as for linear problems, the uniqueness of the solution implies its existence).

The following lemma shows the bound derivatives of the solution for $k=0,1,2,3,4$.

Lemma 2. Let $y_{\varepsilon}$ be the solution of $\left(P_{\varepsilon}\right)$. Then, for $k=0,1,2,3,4$,

$$
\left|y_{\varepsilon}^{(k)}(x)\right| \leq C\left\{1+\varepsilon^{-k}\left(\exp \left(\frac{-c_{0} x}{\varepsilon}\right)+\exp \left(\frac{-c_{1}(l-x)}{\varepsilon}\right)\right)\right\},
$$

for all $x \in \bar{\Omega}$,

where $c_{0}=(1 / 2)\left[\sqrt{a^{2}(0)+4 b(0)}+a(0)\right]$ and $c_{1}=(1 / 2)$ $\left[\sqrt{a^{2}(l)+4 b(l)}-a(l)\right]$.

Proof. For the proof refer [2].

\section{Formulation of the Method}

For small values of $\varepsilon$, the solution $y(x)$ of problems (1)-(3) has in general boundary layers at $x=0$ and $x=l$ (see [2]).

The linear ordinary differential equation (1) cannot, in general, be solved analytically because of the dependence of $a(x)$ and $b(x)$ on the spatial coordinate $x$. We divide the interval $[0,1]$ into $N$ equal parts with constant mesh length $h$. Let $0=x_{0}, x_{1}, \ldots, x_{N}=1$ be the mesh points. Then, we have $x_{i}=i h, i=0,1,2, \ldots, N$. If we consider the interval $x \in(0,1)$ and the coefficients of equation (1) are evaluated at the midpoint of each interval, then we will obtain the differential equation:

$$
\left\{\begin{array}{l}
\varepsilon^{2} y^{\prime \prime}(x)+\varepsilon a(x) y^{\prime}(x)-b(x) y(x)=f(x), \quad x \in \Omega=(0,1), \\
y_{0}=y(0)=\mu_{0} .
\end{array}\right.
$$

In order to evaluate the fitting factor, divide both side of equation (6) by $\mathcal{E}$, and we obtain

$$
\varepsilon y^{\prime \prime}(x)+a(x) y^{\prime}(x)+p(x) y(x)=r(x),
$$

where $p(x)=(-b(x) / \varepsilon)$ and $r(x)=(f(x) / \varepsilon)$.

To find the numerical solution of equation (7), we use the theory applied in the asymptotic method for solving singularly perturbed BVPs. In the considered case, the boundary layer is in the left side of the domain, i.e., near $x=0$. From the theory of singular perturbations given by O'Malley [16] and using Taylor's series expansion for $a(x)$ about $x=0$ and restriction to their first terms, we get the asymptotic solution as

$$
y(x)=y_{0}(x)+\left(\mu_{0}-y_{0}(0)\right) \exp \left(-\frac{a(0)}{\varepsilon} x\right)+O(\varepsilon),
$$

where $y_{0}(x)$ is the solution of the reduced problem (obtained by setting $\varepsilon=0$ ) of equation (7) which is given by

$$
a(x) y_{0}^{\prime}(x)+p(x) y_{0}(x)=r(x), \quad \text { with } y_{0}(1)=\theta_{1} \text {, }
$$

where $\theta_{1}=\mu_{1}+\int_{l_{0}}^{l_{1}} g(x) y(x) \mathrm{d} x$.

Considering $h$ small enough, the discretized form of equation (8) becomes

$$
y(i h)=y_{0}(0)+\left(\mu_{0}-y_{0}(0)\right) \exp \left(-\frac{a(0)}{\mathcal{E}} i h\right),
$$

which simplifies to

$$
y(i h)=y_{0}(0)+\left(\mu_{0}-y_{0}(0)\right) \exp (-i \rho a(0)),
$$

where $\rho=(h / \varepsilon)$ and $h=(1 / N)$.

To handle the effect of the perturbation parameter artificial viscosity, exponentially fitting factor $\sigma(\rho)$ is multiplied on the term containing the perturbation parameter as

$$
\varepsilon \sigma(\rho) y^{\prime \prime}(x)+a(x) y^{\prime}(x)+p(x) y(x)=r(x),
$$

with boundary conditions $y_{0}(0)=\mu_{0}$ and $y(N)=\theta_{1}$.

Next, we consider the difference approximation of equation (7) on a uniform grid $\bar{\Omega}^{N}=\left\{x_{i}\right\}_{i=0}^{N}$ and denote $h=x_{i+1}-x_{i}$. 
For any mesh function $z_{i}$, define the following difference operators:

$$
\begin{aligned}
D^{+} z_{i} & =\frac{z_{i+1}-z_{i}}{h}, \\
D^{-} z_{i} & =\frac{z_{i}-z_{i-1}}{h}, \\
D^{0} z_{i} & =\frac{z_{i+1}-z_{i-1}}{2 h}, \\
D^{+} D^{-} z_{i} & =\frac{z_{i+1}-2 z_{i}+z_{i-1}}{h^{2}} .
\end{aligned}
$$

By applying the central finite difference scheme on equation (12) takes the form

$$
\varepsilon \sigma(\rho)\left(D^{+} D^{-} y\left(x_{i}\right)\right)+a\left(x_{i}\right)\left(D^{0} y\left(x_{i}\right)\right)+p\left(x_{i}\right) y\left(x_{i}\right)=r\left(x_{i}\right) \text {, }
$$

with the boundary conditions $y_{0}(0)=\mu_{0}$ and $y(N)=\theta_{1}$.

Using operator, equation (7) is rewritten as

$$
L^{h} y_{i}=r_{i}
$$

with the boundary conditions $y_{0}=\mu_{0}$ and $y_{N}=\theta_{1}$, where

$$
\begin{aligned}
L^{h} y_{i}= & \varepsilon \sigma(\rho)\left(\frac{y_{i+1}-2 y_{i}+y_{i-1}}{h^{2}}\right)+a\left(x_{i}\right)\left(\frac{y_{i+1}-y_{i-1}}{2 h}\right) \\
& +p\left(x_{i}\right) y_{i}=r_{i} .
\end{aligned}
$$

Multiplying equation (16) by $h$ and considering $h$ small and truncating the term $h\left(r_{i}-p\left(x_{i}\right) y\left(x_{i}\right)\right)$, results

$$
\frac{\varepsilon \sigma(\rho)}{\rho}\left(y_{i+1}-2 y_{i}+y_{i-1}\right)+\frac{a\left(x_{i}\right)}{2}\left(y_{i+1}-y_{i-1}\right)=0 \text {. }
$$

Now using Taylor's series for $y_{i}$ and $y_{i+1}$ up to first term and substituting the results in equation (17) into equation (14) and simplifying, the exponential fitting factor is obtained as

$$
\sigma(\rho)=\frac{\rho a(0)}{2} \operatorname{coth}\left(\frac{\rho a(0)}{2}\right)
$$

Similarly, by following the above mentioned procedure, the fitting factor for the right layer becomes

$$
\sigma(\rho)=\frac{\rho a(1)}{2} \operatorname{coth}\left(\frac{\rho a(1)}{2}\right)
$$

Assume that $\bar{\Omega}^{N}$ denote partition of $[0,1]$ into $N$ subintervals such that $0=x_{0}<x_{1}<\cdots<x_{N}=1$ with $x_{i}=i h, h=(1 / N), i=0,1,2, \ldots, N$.

Case 1. For the left layer, consider equation (6) on the domain $\Omega=(0,1)$ which is given by

$$
\varepsilon^{2} y^{\prime \prime}(x)+\varepsilon a(x) y^{\prime}(x)-b(x) y(x)=f(x) .
$$

Hence, the required finite difference scheme becomes

$$
\left(\frac{\varepsilon^{2} \sigma(\rho)}{h^{2}}\right) y_{i-1}+\left(\frac{-2 \varepsilon^{2} \sigma(\rho)}{h^{2}}-\frac{\varepsilon a\left(x_{i}\right)}{h}-b\left(x_{i}\right)\right) y_{i}+\left(\frac{\varepsilon^{2} \sigma(\rho)}{h^{2}}+\frac{\varepsilon a\left(x_{i}\right)}{2 h}\right) y_{i+1}=f_{i} \text {, }
$$

for $i=0,1,2, \ldots, N$.

The numerical scheme in equation (21) can be written in three-term recurrence relation as

$$
E_{i} y_{i-1}+F_{i} y_{i}+G_{i} y_{i+1}=H_{i}, \quad i=1,2, \ldots, N,
$$

where $E_{i}=\left(\varepsilon^{2} \sigma / h^{2}\right), F_{i}=\left(-2 \varepsilon^{2} \sigma / h^{2}\right)-\left(\varepsilon a_{i} / h\right)-b_{i}, G_{i}=$ $\left(\varepsilon^{2} \sigma / h^{2}\right)+\left(\varepsilon a_{i} / 2 h\right)$, and $H_{i}=f_{i}$.
Case 2. For the right layer, consider equation (6) on the domain $\Omega=(0,1)$ which is given by

$$
\varepsilon^{2} y^{\prime \prime}(x)+\varepsilon a(x) y^{\prime}(x)-b(x) y(x)=f(x) .
$$

Hence, the required finite difference scheme becomes

$$
\left(\frac{\varepsilon^{2} \sigma(\rho)}{h^{2}}-\frac{\varepsilon a\left(x_{i}\right)}{h}\right) y_{i-1}+\left(\frac{-2 \varepsilon^{2} \sigma(\rho)}{h^{2}}+\frac{\varepsilon a\left(x_{i}\right)}{h}-b\left(x_{i}\right)\right) y_{i}+\left(\frac{\varepsilon^{2} \sigma(\rho)}{h^{2}}\right) y_{i+1}=f_{i},
$$

for $i=0,1,2, \ldots, N$.

The numerical scheme in equation (24) can be written in three-term recurrence relation as

$$
E_{i} y_{i-1}+F_{i} y_{i}+G_{i} y_{i+1}=H_{i}, \quad i=1,2, \ldots, N,
$$

where $E_{i}=\left(\varepsilon^{2} \sigma / h^{2}\right)-\left(\varepsilon a_{i} / h\right), \quad F_{i}=\left(-2 \varepsilon^{2} \sigma / h^{2}\right)+\left(\varepsilon a_{i} / h\right)-$ $b_{i}, G_{i}=\left(\varepsilon^{2} \sigma / h^{2}\right)$, and $H_{i}=f_{i}$,
For $i=N$, we considered Simpson's rule, i.e., treating the integral boundary condition using Simpson's rules. Suppose $g(x) y(x)$ is a function defined on the interval $[0, l]$, and let $\left\{x_{i}\right\}_{i=0}^{2 N}$ be a uniform partition of $[0, l]$ with step length $h$.

The composite Simpson's rule approximates the integral of $g(x) y(x)$ is given by 


$$
\int_{l_{0}}^{l_{1}} g(x) y(x) \mathrm{d} x=\frac{h}{3}\left(g\left(l_{0}\right) y\left(l_{0}\right)+g\left(l_{1}\right) y\left(l_{1}\right)+2 \sum_{i=1}^{2 N-1} g\left(x_{2 i}\right) y\left(x_{2 i}\right)+4 \sum_{i=1}^{2 N} g\left(x_{2 i-1}\right) y\left(x_{2 i-1}\right)\right) .
$$

Substituting equation (26) into equation (3) gives

$$
y(l)-\frac{h}{3}\left(g\left(l_{0}\right) y\left(l_{0}\right)+g\left(l_{1}\right) y\left(l_{1}\right)+2 \sum_{i=1}^{2 N-1} g\left(x_{2 i}\right) y\left(x_{2 i}\right)+4 \sum_{i=1}^{2 N} g\left(x_{2 i-1}\right) y\left(x_{2 i-1}\right)\right)=\mu_{1} .
$$

Since $y(0)=\mu_{0}$, from equations (2) and (27), we obtain:

$$
-\frac{4 h}{3} \sum_{i=1}^{2 N} g\left(x_{2 i-1}\right) y\left(x_{2 i-1}\right)-\frac{2 h}{3} \sum_{i=1}^{2 N-1} g\left(x_{2 i}\right) y\left(x_{2 i}\right)+y(l)-\frac{h}{3} g\left(l_{1}\right) y\left(l_{1}\right)=\mu_{1}+\frac{h}{3} g\left(l_{0}\right) y\left(l_{0}\right) \text {. }
$$

Therefore, the problem in equation (1) with given boundary condition in equations (2) and (3) can be solved using the scheme equations (22), (25), and (28) which forms $N \times N$ system of algebraic equations.

\section{Uniform Convergence Analysis}

In this section, we need to show the discrete scheme in equations (22), (25), and (28) satisfy the discrete minimum principle and uniform convergence.

Lemma 3 (discrete minimum principle). Let $v_{i}$ be any mesh function that satisfies $v_{0} \geq 0, v_{N} \geq 0$, and $L^{h} v_{i} \leq 0, i=1,2, \ldots, N-1$, then $v_{i} \geq 0, i=0,1,2, \ldots, N$.

Proof. The proof is obtained by contradiction. Let $j$ be such that $v_{j}=\min _{i} v_{i}$ and suppose that $v_{j}<0$. Clearly, $j \notin\{0, N\}, v_{j+1}-v_{j} \geq 0$ and $v_{j}-v_{j-1} \leq 0$.

Therefore,

$$
\begin{aligned}
L^{h} v_{j} & =\frac{\varepsilon}{h^{2}}\left(v_{j+1}-2 v_{j}+v_{j-1}\right)+\frac{a_{j}}{h}\left(v_{j+1}-v_{j}\right)+p_{j} v_{j} \\
& =\frac{\varepsilon}{h^{2}}\left[\left(v_{j+1}-v_{j}\right)-\left(v_{j}-v_{j-1}\right)\right]+\frac{a_{j}}{h}\left(v_{j+1}-v_{j}\right)+p_{j} v_{j} \\
& \geq 0
\end{aligned}
$$

where the strict inequality holds if $v_{j+1}-v_{j}>0$. This is a contradiction, and therefore $v_{j} \geq 0$. Since $j$ is arbitrary, we have $v_{i} \geq 0, i=0,1,2, \ldots, N$.

We proved above the discrete operator $L^{h}$ satisfy the minimum principle. Next, we analyze the uniform convergence analysis. Let us define the forward, backward, and second order finite difference operators as follows:

$$
\begin{aligned}
D^{+} v_{j} & =\frac{v_{j+1}-v_{j}}{h}, \\
D^{-} v_{j} & =\frac{v_{j}-v_{j-1}}{h}, \\
\delta^{2} v_{j} & =D^{+} D^{-} v_{j}=\frac{D^{+} v_{j}-D^{-} v_{j}}{h} .
\end{aligned}
$$

Theorem 1. Let $y\left(x_{i}\right)$ and $y_{i}$ be, respectively, the exact solution of (1)-(3) and numerical solutions of equation (15). Then, for sufficiently large $N$, the following parameter uniform error estimate holds:

$$
\sup _{0<\varepsilon \leq 1}\left\|y\left(x_{i}\right)-y_{i}\right\| \leq C N^{-2}
$$

Proof. Let us consider the local truncation error defined as

$$
\begin{aligned}
L^{h}\left(y\left(x_{i}\right)-y_{i}\right)= & \varepsilon \sigma(\rho)\left(\frac{\mathrm{d}^{2}}{\mathrm{~d} x^{2}}-D^{+} D^{-}\right) y\left(x_{i}\right) \\
& +a\left(x_{i}\right)\left(\frac{\mathrm{d}}{\mathrm{d} x}-D^{+}\right) y\left(x_{i}\right)
\end{aligned}
$$

where $\varepsilon \sigma(\rho)=a(0)\left(N^{-1} / 2\right) \operatorname{coth}\left(a(0)\left(N^{-1} / 2 \varepsilon\right)\right) \quad$ since $\rho=\left(N^{-1} / \varepsilon\right)$. In our assumption $\varepsilon \leq h=N^{-1}$.

By considering $N$ is fixed and taking the limit for $\varepsilon \longrightarrow 0$, we obtain the following:

$$
\lim _{\varepsilon \longrightarrow 0} \varepsilon \sigma(\rho)=\lim _{\varepsilon \longrightarrow 0} a(0) \frac{N^{-1}}{2} \operatorname{coth}\left(a(0) \frac{N^{-1}}{2 \varepsilon}\right)=C N^{-1}
$$

From Taylor series expansion, the bound for the difference becomes 


$$
\left\{\begin{array}{l}
\left\|\left(\frac{\mathrm{d}^{2}}{\mathrm{~d} x^{2}}-D^{+} D^{-}\right) y\left(x_{i}\right)\right\| \leq C N^{-3}\left\|\frac{\mathrm{d}^{4}\left(y\left(x_{i}\right)\right)}{\mathrm{d} x^{4}}\right\|, \\
\left\|\left(\frac{\mathrm{d}}{\mathrm{d} x}-D^{+}\right) y\left(x_{i}\right)\right\| \leq C N^{-2}\left\|\frac{\mathrm{d}^{3}\left(y\left(x_{i}\right)\right)}{\mathrm{d} x^{3}}\right\|,
\end{array}\right.
$$

where $\left\|\left(\mathrm{d}^{k}\left(y\left(x_{i}\right)\right)\right) /\left(\mathrm{d} x^{k}\right)\right\|=\sup _{x_{i} \in\left(x_{0}, x_{N}\right)}\left(\left(\mathrm{d}^{k} y\left(x_{i}\right)\right) /\left(\mathrm{d} x^{k}\right)\right)$, $k=3,4$.

Now using the bounds and the assumption $\varepsilon \leq N^{-1}$, equation (32) reduces to

$$
\begin{aligned}
\left\|L^{h}\left(y\left(x_{i}\right)-y_{i}\right)\right\| & =\left\|\varepsilon \sigma(\rho)\left(\frac{\mathrm{d}^{2}}{\mathrm{~d} x^{2}}-D^{+} D^{-}\right) y\left(x_{i}\right)+a\left(x_{i}\right)\left(\frac{\mathrm{d}}{\mathrm{d} x}-D^{+}\right) y\left(x_{i}\right)\right\| \\
& \leq\left\|\varepsilon \sigma(\rho)\left(\frac{\mathrm{d}^{2}}{\mathrm{~d} x^{2}}-D^{+} D^{-}\right) y\left(x_{i}\right)\right\|+\left\|a\left(x_{i}\right)\left(\frac{\mathrm{d}}{\mathrm{d} x}-D^{+}\right) y\left(x_{i}\right)\right\| \\
& \leq C N^{-3}\left\|\frac{\mathrm{d}^{4}\left(y\left(x_{i}\right)\right)}{\mathrm{d} x^{4}}\right\|+C N^{-2}\left\|\frac{\mathrm{d}^{3}\left(y\left(x_{i}\right)\right)}{\mathrm{d} x^{3}}\right\| .
\end{aligned}
$$

Here, the target is to show the scheme convergence independent on the number of mesh points.
By using the bounds for the derivatives of the solution in Lemma 2, we obtain

$$
\begin{aligned}
\left\|L^{h}\left(y\left(x_{i}\right)-y_{i}\right)\right\| \leq & C N^{-3}\left\|\frac{\mathrm{d}^{4}\left(y\left(x_{i}\right)\right)}{\mathrm{d} x^{4}}\right\|+C N^{-2}\left\|\frac{\mathrm{d}^{3}\left(y\left(x_{i}\right)\right)}{\mathrm{d} x^{3}}\right\| \\
\leq & C N^{-3}\left\{1+\varepsilon^{-4}\left(\exp \left(\frac{-c_{0} x}{\varepsilon}\right)+\exp \left(\frac{-c_{1}(l-x)}{\varepsilon}\right)\right)\right\} \\
& +C N^{-2}\left\{1+\varepsilon^{-3}\left(\exp \left(\frac{-c_{0} x}{\varepsilon}\right)+\exp \left(\frac{-c_{1}(l-x)}{\varepsilon}\right)\right)\right\} \\
\leq & C N^{-2}\left\{1+\varepsilon^{-4}\left(\exp \left(\frac{-c_{0} x}{\varepsilon}\right)+\exp \left(\frac{-c_{1}(l-x)}{\varepsilon}\right)\right)\right\}, \text { since } \varepsilon^{-4} \geq \varepsilon^{-3} .
\end{aligned}
$$

Most of the time during analysis, one encounters with exponential terms involving $\varepsilon$ divided by the power function in $\varepsilon$ which are always the main cause of worry. For their careful consideration while proving the $\varepsilon$-uniform convergence, we prove for the right layer case as follows.

Lemma 4. For a fixed mesh and for $\varepsilon \longrightarrow 0$, it holds:

$$
\lim _{\varepsilon \longrightarrow 0} \max _{1 \leq j \leq N-1}\left(\frac{\exp \left(\left(-c_{0} x\right) / \varepsilon\right)}{\varepsilon^{3}}+\frac{\exp \left(\left(-c_{1}(l-x)\right) / \varepsilon\right)}{\varepsilon^{3}}\right)=0, \quad m=1,2,3, \ldots
$$

where $x_{j}=j h, h=(1 / N), \forall j=1,2, \ldots, N-1$.

Proof. Consider the partition $[0,1]:=\left\{0=x_{0}<x_{1}<\cdots<\right.$ $\left.x_{N-1}<x_{N}=1\right\}$ for the interior grid points, we have

$$
\begin{aligned}
\max _{1 \leq j \leq N-1} \frac{\exp \left(\left(-c_{0} x_{j}\right) / \varepsilon\right)}{\varepsilon^{m}} \leq & \frac{\exp \left(\left(-c_{0} x_{1}\right) / \varepsilon\right)}{\varepsilon^{m}}=\frac{\exp \left(\left(-c_{0} h\right) / \varepsilon\right)}{\varepsilon^{m}} \\
\max _{1 \leq j \leq N-1} \frac{\exp \left(\left(-c_{1}\left(1-x_{j}\right)\right) / \varepsilon\right)}{\varepsilon^{m}} \leq & \frac{\exp \left(\left(-c_{1}\left(1-x_{N-1}\right)\right) / \varepsilon\right)}{\varepsilon^{m}}=\frac{\exp \left(\left(-c_{1} h\right) / \varepsilon\right)}{\varepsilon^{m}}, \\
& \cdot\left(\text { as } x_{1}=h, 1-x_{N-1}=1-(N-1) h=h\right) .
\end{aligned}
$$


TABLE 1: Maximum absolute errors and rates of convergence for our example.

\begin{tabular}{lccccc}
\hline$\varepsilon$ & $N=32$ & $N=64$ & $N=128$ & $N=256$ & $N=512$ \\
\hline $10^{-4}$ & $6.2800 e-04$ & $1.5350 e-04$ & $3.7899 e-05$ & $9.4122 e-06$ & 2.0049 \\
& 2.0325 & 2.0180 & 2.0096 & $9.5046 e-06$ & $2.3501 e-06$ \\
$10^{-8}$ & $6.3295 e-04$ & $1.5487 e-04$ & $3.8258 e-05$ & 2.0047 & $2.3685 e-06$ \\
& 2.0310 & 2.0172 & $3.8258 e-05$ & $2.5046 e-06$ & 2.0047 \\
$10^{-12}$ & $6.3295 e-04$ & $1.5487 e-04$ & 2.0091 & $9.5046 e-06$ & 2.0047 \\
& 2.0310 & 2.0172 & $3.8258 e-05$ & 2.0091 & $9.5046 e-06$ \\
$10^{-16}$ & $6.3295 e-04$ & $1.5487 e-04$ & $3.8258 e-05$ & 2.0047 \\
& 2.0310 & $1.5487 e-04$ & 2.009172 & $2.3685 e-06$ \\
$10^{-20}$ & $6.3295 e-04$ & 2.0172 & $3.8258 e-05$ & $2.5046 e-06$ \\
& 2.0310 & $1.5487 e-04$ & 2.0091 & 2.0047 \\
\hline$E^{h}$ & $6.3295 e-04$ & 2.0172 & & $2.3685 e-06$ \\
\hline$R^{h}$ & 2.0310 & &
\end{tabular}

TABle 2: Comparison of maximum absolute errors and rates of convergence of our example.

\begin{tabular}{lcccc}
\hline & $N=16$ & $N=32$ & $N=16$ & $N=32$ \\
& \multicolumn{2}{c}{ Present method } & \multicolumn{2}{c}{ Method in [2] } \\
\hline $10^{-3}$ & $2.4362 e-03$ & $5.7378 e-04$ & $1.5304 e-02$ & $7.8100 e-03$ \\
& 2.0861 & & 0.97 & \\
$10^{-5}$ & $2.6259 e-03$ & $6.3247 e-04$ & $1.5304 e-02$ & $7.8810 e-03$ \\
& 2.0537 & & 0.96 & \\
$10^{-7}$ & $2.6276 e-03$ & $6.3295 e-04$ & $1.5304 e-02$ & $7.8810 e-03$ \\
& 2.0536 & & 0.96 & \\
\hline$E^{h}$ & $2.6276 e-03$ & $6.3295 e-04$ & $1.5304 e-02$ & $7.8810 e-03$ \\
$R^{h}$ & 2.0536 & & 0.96 \\
\hline
\end{tabular}

The repeated application of L'Hospital's rule gives

$$
\begin{aligned}
\lim _{\varepsilon \longrightarrow 0} \frac{\exp ((-c h) / \varepsilon)}{\varepsilon^{m}} & =\lim _{\sigma=(1 / \varepsilon) \longrightarrow \infty} \frac{\sigma^{m}}{\exp (c h \sigma)} \\
& =\lim _{\sigma=(1 / \varepsilon) \longrightarrow \infty} \frac{m !}{(c h)^{m} \exp (c h \sigma)}=0 .
\end{aligned}
$$

This complete the proof.

Theorem 2. Under the hypothesis of boundness of discrete solution (i.e., it satisfies the discrete minimum principle), Lemma 4 and Theorem 1, the discrete solution satisfy the following bound:

$$
\sup _{0 \leq \varepsilon \leq 1} \max _{i}\left|y_{i}-Y_{i}\right| \leq C N^{-1} .
$$

Proof. Results from boundness of solution, Lemma 4 and Theorem 1, give the required estimates.

5.1. Richardson Extrapolation. This technique is acceleration technique which involves combination of two computed approximations of a solution. The combination goes out to be an improved approximation. From the local truncation term, we have

$$
\left|y\left(x_{i}\right)-y_{i}\right| \leq C(h)
$$

where $y\left(x_{i}\right)$ and $y_{i}$ are exact and approximate solutions, respectively, and $C$ is constant free from mesh size $h$.

Let $\Omega^{4 N}$ be the mesh found by dividing each mesh interval in $\Omega^{2 N}$ and symbolize the calculation of the solution on $\Omega^{4 N}$ by $\bar{y}_{i}$. Consider equation (41) works for any $h \neq 0$, which implies

$$
y\left(x_{i}\right)-y_{i} \leq C(h)+R^{2 N}, \quad x_{i} \in \Omega^{2 N} .
$$

So that it works for any $(h / 2) \neq 0$ yielding

$$
y\left(x_{i}\right)-\bar{y}_{i} \leq C\left(\frac{h}{2}\right)+R^{4 N}, \quad x_{i} \in \Omega^{4 N},
$$

where the remainders $R^{2 N}$ and $R^{4 N}$ are $O\left(h^{2}\right)$. Combination of inequalities in equations (42) and (43) leads to $y\left(x_{i}\right)$ $\left(2 \bar{y}_{i}-y_{i}\right) \approx O\left(h^{2}\right)$ which proposes that

$$
\left(y_{i}\right)^{\mathrm{ext}}=2 \bar{y}_{i}-y_{i}
$$

is also a rough calculation of $y\left(x_{i}\right)$. By means of this approximation to estimate the truncation error, we obtain

$$
\left|y\left(x_{i}\right)-\left(y_{i}\right)^{\mathrm{ext}}\right| \leq C\left(h^{2}\right),
$$

where $C$ is free of mesh size $h$. Thus, using Richardson extrapolation first-order convergent method is accelerated into second-order convergent as provided in (45). Thus, we can say that the proposed method is second-order convergent.

\section{Numerical Example and Results}

To validate the established theoretical results, we perform numerical experiments using the model problem of the form in equations (1)-(3).

Example 1. Consider the model singularly perturbed boundary value problem:

$$
\varepsilon^{2} y^{\prime \prime}+2 \varepsilon(x+1) y^{\prime}-\left(-x^{2}-2 x+4\right) y=-\left(e^{x}+e^{x^{2}}\right)
$$

which subject to the conditions

$$
\begin{aligned}
y(0) & =1, \\
y(1)-\int_{0.5}^{1} \cos (x) y(x) \mathrm{d} x & =0 .
\end{aligned}
$$




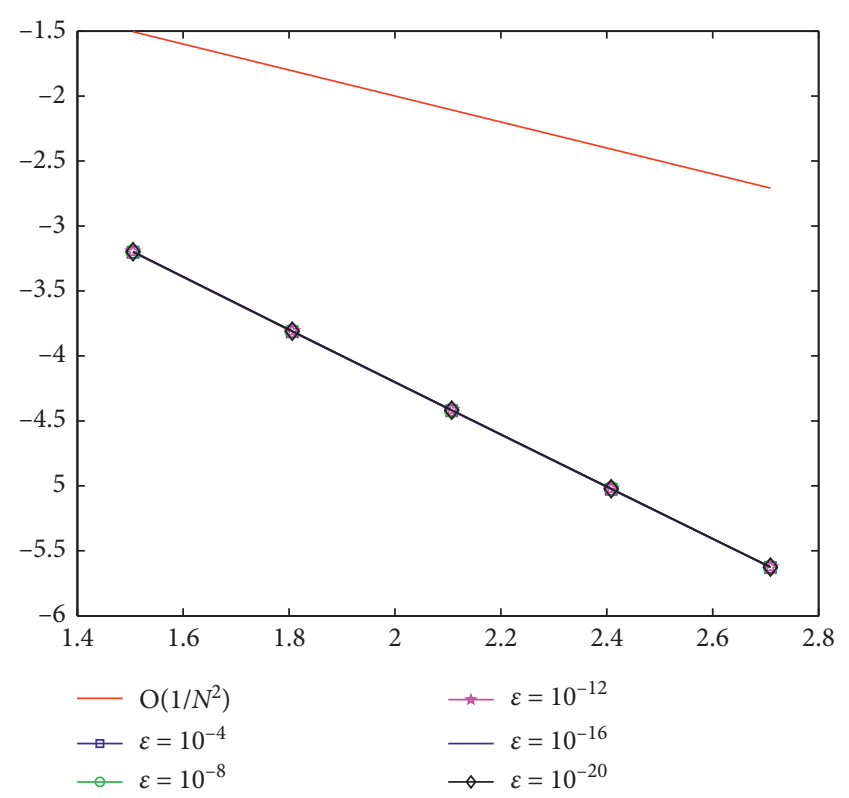

FIGURE 1: $\varepsilon$-uniform convergence with fitted operator FDM in loglog scale for our example.

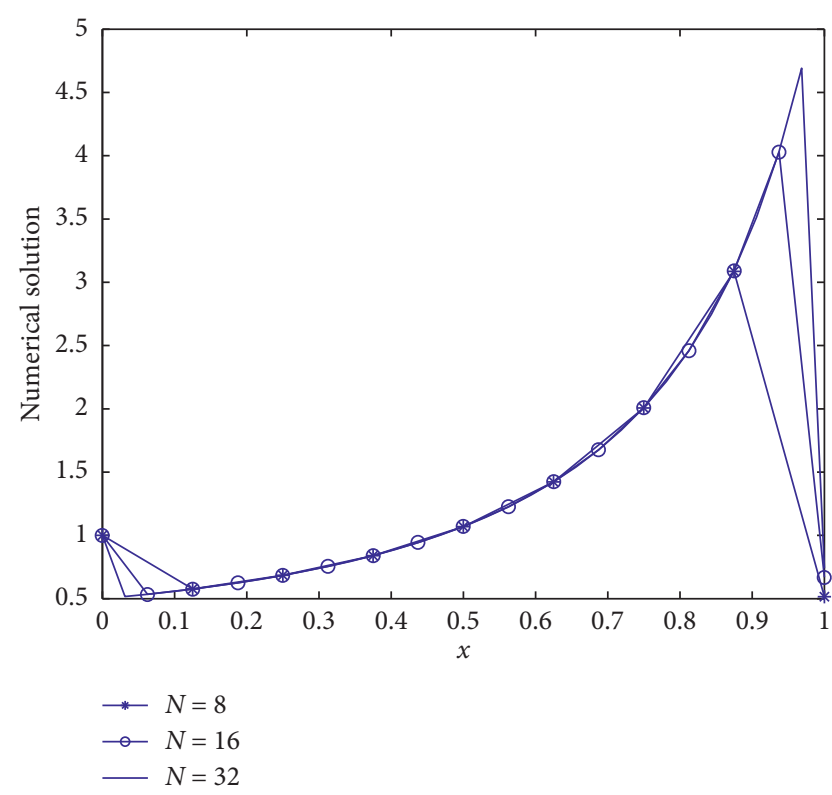

FIGURE 2: The numerical solution for various values of $N$ and $\varepsilon=10^{-3}$.

We have $y_{j} \equiv y_{j}^{h}$ (the approx. solution obtained via the present method) for different values of $h$ and $\varepsilon$, since the exact solution is not available; the maximum errors (denoted by $E_{\varepsilon}^{h}$ ) are evaluated using the formula given by the double mesh principle [17]:

$$
E_{\varepsilon}^{h}:=\max _{0 \leq j \leq N}\left|y_{j}^{h}-y_{2 j}^{2 h}\right| .
$$

Furthermore, we will tabulate the errors:

$$
E^{h}=\max _{0<\varepsilon \leq 1} E_{\varepsilon}^{h} \text {. }
$$

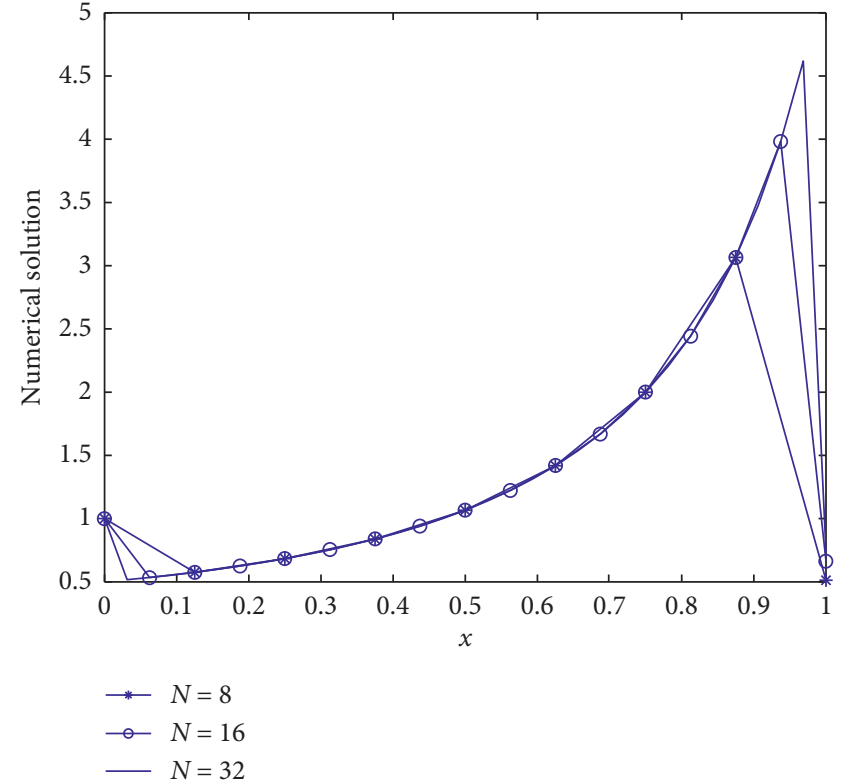

Figure 3: The numerical solution for various values of $N$ and $\varepsilon=10^{-5}$.

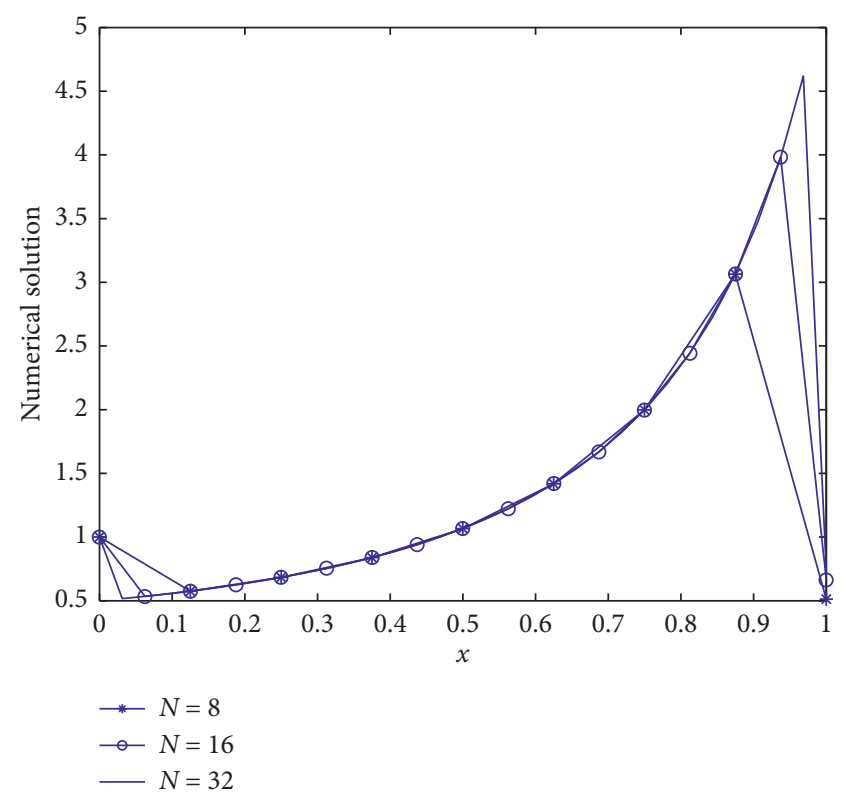

FIgURE 4: The numerical solution for various values of $N$ and $\varepsilon=10^{-7}$.

The numerical rates of convergence are computed using the following formula [17]:

$$
r_{\varepsilon}^{h}:=\log _{2}^{E_{\varepsilon}^{h}}-\log _{2}^{E_{\varepsilon}^{h / 2}}
$$

and the numerical rate of " $\varepsilon$-uniform convergence" is computed using

$$
R^{h}=\log _{2}^{E^{h}}-\log _{2}^{E^{h / 2}} .
$$




\section{Discussion and Conclusion}

This study introduces the uniformly convergent numerical method based on the exponential fitted operator method for solving singularly perturbed boundary value problems with integral boundary conditions. The behavior of the continuous solution of the problem is studied and the derivatives of the solution are also bounded. The numerical scheme is developed on a uniform mesh. The integral boundary condition is treated using numerical integration techniques, namely, Simpson's rule; the results are compared accordingly. The stability of the developed scheme is established and its uniform convergence is proved. To validate the applicability of the method, a model problem/example is considered for numerical experimentation for different values of the perturbation parameter and mesh points. The numerical results are tabulated in terms of maximum absolute errors, numerical rate of convergence, and uniform errors (see Tables 1 and 2) and compared with the results of the previously developed numerical methods existing in the literature (Table 2). Furthermore, the $\varepsilon$-uniform convergence of the method is shown by the log-log plot of the $\mathcal{E}$-uniform error (Figure 1) and the numerical solution for various values of $N$ and $\varepsilon$ are given (see Figures 2-4). Unlike other fitted operator finite difference methods constructed in standard ways, the method that we presented in this paper is fairly simple to construct.

\section{Data Availability}

No data were used to support this study.

\section{Conflicts of Interest}

The authors declare that they have no conflicts of interest.

\section{Authors' Contributions}

HGD proposed the main idea of this paper. HGD and GFD prepared the manuscript and performed all the steps of the proofs in this research. Both authors contributed equally and significantly in writing this paper. Both authors read and approved the final manuscript.

\section{Acknowledgments}

The authors wish to express their thanks to Jimma University, College of Natural Sciences, for financial support and the authors of the literature for the provided scientific aspects and idea for this work.

\section{References}

[1] M. Benchohra, S. Hamani, and J. J. Nieto, "The method of upper and lower solutions for second order differential inclusions with integral boundary conditions," Rocky Mountain Journal of Mathematics, vol. 40, no. 1, pp. 13-26, 2010.

[2] M. Cakr and G. M. Amralyev, "A finite difference method for the singularly perturbed problem with nonlocal boundary condition," Applied Mathematics and Computation, vol. 160, no. 2, pp. 539-549, 2005.
[3] A. Belarbi and M. Benchohra, "Existence results for nonlinear boundary value problems with integral boundary conditions," Electronic Journal of Differential Equations, vol. 6, pp. 1-10, 2005.

[4] A. Belarbi, M. Benchohra, and A. Quahab, "Multiple positive solutions for nonlinear boundary value problems with integral boundary conditions," Archiv der Mathematik, vol. 44, no. 1, pp. 1-7, 2008.

[5] M. Benchohra, F. Berhoun, and J. Henderson, "Multiple positive solutions for impulsive boundary value problem with integral boundary conditions," Mathematical Sciences Research Journal, vol. 11, no. 12, pp. 614-626, 2007.

[6] B. Ahmad, R. A. Khan, and S. Sivasundaram, "Generalized quasilinearization method for a first order differential equation with integral boundary condition," Dynamics of Continuous, Discrete and Impulsive Systems, vol. 12, pp. 289-296, 2005.

[7] J. Du and M. Cui, "Solving the forced duffing equation with integral boundary conditions in the reproducing kernel space," International Journal of Computer Mathematics, vol. 87, no. 9, pp. 2088-2100, 2010.

[8] G. M. Amiraliyev and B. Ylmaz, "Finite difference method for singularly perturbed differential equations with integral boundary condition," International Journal of Computer Mathematics, vol. 22, no. 1, pp. 11-10, 2014.

[9] E. P. Doolan, J. J. H. Miller, and W. H. A. Schilders, Uniform Numerical Methods for Problems with Initial and Boundary Layers, Boole-Press, Dublin, Ireland, 1980.

[10] P. A. Farrell, A. F. Hegarty, J. J. H. Miller, E. ORiordan, and G. I. Shishkin, Robust Computational Techniques for Boundary Layers, Charman and Hall/CRC, Boca Raton, FL, USA, 2002.

[11] M. Kudu and G. M. Amiraliyev, "Finite difference method for a singularly perturbed differential equations with integral boundary condition," International Journal of Mathematics and Computation, vol. 26, no. 3, pp. 72-78, 2015.

[12] M. Cakir, "A numerical study on the difference solution of singularly perturbed semilinear problem with integral boundary condition," Mathematical Modelling and Analysis, vol. 21, no. 5, pp. 644-658, 2016.

[13] M. Kudu, I. Amirali, and G. M. Amiraliyev, "Uniform numerical approximation for parameter dependent singularly perturbed problem with integral boundary condition," Miskolc Mathematical Notes, vol. 19, no. 1, pp. 337-353, 2018.

[14] E. Sekar and A. Tamilselvan, "Singularly perturbed delay differential equations of convection diffusion type with integral boundary condition," Journal of Applied Mathematics and Computing, vol. 59, no. 1-2, pp. 701-722, 2019.

[15] G. Habtamu and F. Gemechis, "Exponentially fitted finite difference method for singularly perturbed delay differential equations with integral boundary condition," International Journal of Engineering and Applied Sciences, vol. 11, no. 4, pp. 476-493, 2019.

[16] R. E. O'Malley, Singular Perturbation Methods for Ordinary Differential Equations, Springer, Berlin, Germany, 1991.

[17] J. J. H. Miller, E. ORiordan, and G. I. Shishkin, Fitted Numerical Methods for Singular Perturbation Problems, World Scientific, Singapore, 2012. 